This is the post print version of the article, which has been published in Journal of Plastic Surgery and Hand Surgery. 2018, 52(1), 1-6. http://dx.doi.org/10.1080/2000656X.2017.1310734.

\title{
Survey of hand surgeons' and therapists' perceptions of the benefit of common surgical procedures of the hand
}

\author{
Olli V. Leppänen ${ }^{1,2}$, Jarkko Jokihaara ${ }^{1}$, Esa Jämsen ${ }^{2,3}$, Teemu Karjalainen ${ }^{4}$ \\ ${ }^{1}$ Department of hand surgery, Tampere University Hospital, Tampere, Finland \\ ${ }^{2}$ School of Medicine, University of Tampere, Tampere, Finland \\ ${ }^{3}$ Coxa Hospital for Joint Replacement, Tampere, Finland \\ ${ }^{4}$ Department of Surgery, Central Finland Central Hospital, Jyväskylä, Finland
}

\section{Corresponding author:}

Olli V. Leppänen, M.D., Ph.D.

Tampere University Hospital

Department of Hand and Microsurgery

P.O. Box 2000, 33521 Tampere

Finland

Email: olli.v.leppanen@uta.fi

Phone: +358-3-311611

Acknowledgements: Mrs. Piia Hautala is thanked for her help in contacting the members of the Finnish Society for Hand Therapy.

Conflict of interest: The authors declared no potential conflict of interest with respect to the research, authorship, and/or publication of this article.

Funding: The authors received no financial support for the research, authorship, and/or publication of this article. 


\section{Survey of hand surgeons' and therapists' perceptions of the benefit of common surgical procedures of the hand}

Objective: The objective of this survey was to 1) study if surgeons' perceptions of the benefit of six surgical procedures differ if they consider themselves as patients instead of treating a patient, 2) to evaluate the role of five predetermined factors that may influence decision-making, and 3) to assess how uniformly hand surgeons and hand therapists perceive the benefits of the surgical treatments.

Methods: The members of the national societies for Hand Surgery and Hand Therapy were asked to participate in the survey. Six patient cases with hand complaint (carpal tunnel syndrome, flexor tendon injury, dorsal wrist ganglion, thumb amputation, boxer's fracture, and mallet fracture) and a proposed operative procedure were presented and the respondents rated the procedures in terms of the expected benefit. Half of the surgeons were advised to consider themselves as patients when filling out the survey.

Results: A survey was completed by 56 surgeons (61\%) and 59 therapists (20\%). Surgeons who considered themselves as patients had less confident perception on the benefit of carpal tunnel release compared with surgeons, who considered treating patients. Hand surgeons and hand therapists had similar perception of the benefits of surgery. The expected functional result was regarded as the most important factor directing the decision about the treatment.

Conclusions: Surgeons tended to be more unanimous in their opinions in cases, where there is limited evidence on treatment effect. The agreement between surgeons and therapists implies that the clinical perspectives are similar and probably reflect the reality well.

Keywords: survey; questionnaire; carpal tunnel syndrome; hand; surgery; decision-making

\section{Introduction}

Evidence-based surgery is an integration of the best research evidence with clinical expertise and patient values [1]. Previously, it has been shown that surgeons choose riskier but potentially more effective treatments for themselves than they would 
recommend to their patients [2]. On the other hand, it has been shown that surgeons are slightly more likely to recommend surgery for a patient than for themselves in a similar situation [3]. If the surgeon is making the decision rationally - based on the current evidence and local treatment policies - this kind of inconsistency should not exist.

Hand therapy is a crucial part of the treatment of various hand conditions and a hand therapist undeniably spends more time with most of the patients than a surgeon. However, hand therapists are seldom taking part in the decision-making concerning surgical treatment, which is done by the surgeon and the patient. Thus, hand therapists might be able to evaluate the effectiveness of different surgical procedures of the hand more objectively than hand surgeons.

The objectives of this study were 1) to investigate if there is difference in decision-making when surgeons are treating a patient or considering themselves as patients in six common hand surgical conditions, 2) to evaluate the role of five predetermined factors (functional result, cosmetic appearance, cost of treatment, length of hospital stay, and length of sick leave) that may influence decision-making, and 3) to assess how uniformly hand surgeons and hand therapists perceive the benefits of the surgical treatments.

\section{Methods}

\section{Study participants}

In this cross-sectional survey, the members of the national Society for the Surgery of the Hand and Society for Hand Therapy were contacted via e-mail containing a hyperlink to an electronic study questionnaire form. The responses were collected during a 1-month period (March - April, 2016) and biweekly reminders were mailed to all members. 


\section{Study questionnaire}

There were two types of questionnaires: 1) A randomized half of the hand surgeons and residents, and all hand therapists received a questionnaire that described six patient cases with a hand complaint (carpal tunnel syndrome, flexor tendon injury, dorsal wrist ganglion, thumb amputation, boxer's fracture, and mallet fracture), and 2) the other half of the hand surgeons and residents received a questionnaire that asked the recipient to imagine him- or herself having the same six described hand complaints. The randomization was carried out using a random number generator software. Each responder was only shown one questionnaire and he or she was unaware of the other questionnaire. All cases were accompanied by a proposal for surgical treatment and a set of questions related to the choice of treatment. The patient cases are described in Tables 1-6 and Figures 1-3.

The cases included common traumatic and non-traumatic conditions, which have relative operative indications (i.e. the non-operative treatment would not probably result in catastrophic clinical outcome, and either conservative or operative treatment would not currently be considered malpractice). For five of the conditions, the current evidence for preferred treatment is weak, whereas carpal tunnel syndrome is the most thoroughly studied condition of the hand $[1,4]$. The patient cases were simplified in terms of medical history (i.e., no descriptions of previous injuries, medications, surgical operations, and diseases). To eliminate the bias arising from the potentially different age and gender distribution between the respondents and described patient cases, the patient cases were adjusted to match the respondents' age and gender. To avoid untypical patient descriptions if the respondent was younger than an average patient would be, degenerative conditions (e.g., basal thumb arthrosis, triangular fibrocartilage complex tears) were not addressed in this study. The case descriptions and the questionnaires 
were in Finnish. For this report, the text was translated to English. To ensure the validity of the translation process, an independent translator translated the English version back to Finnish. The two Finnish versions were then compared for similarity.

\section{Outcome variables}

The demographic data of the study participants (gender, age, position: surgeon/resident/therapist) were recorded. The primary outcome variable was the perception of the benefit of the proposed surgical procedure in the described cases. The respondents were asked to rate the operation from "Very harmful" to "Very beneficial" on a 11-step scale $(-5 \ldots+5)$ regarding the question: "How beneficial do you find the proposed surgical treatment for your patient?" or "How beneficial do you find the proposed surgical treatment to you as a patient?". Zero was labelled as "Neither beneficial nor harmful". In addition, the respondents were asked to rate how the following factors influenced their decision-making on a 5-step scale from "Not at all important" to "Very important": 1) functional result, 2) cosmetic appearance, 3) cost of treatment, 4) length of hospital stay, and 5) length of sick leave. The rationale behind these five predetermined factors was the authors' personal experience about factors that may have an influence on clinical decision making.

\section{Statistical analysis}

Analysis of variance (ANOVA) was used for the comparisons between the groups. The statistical significance was set to $\mathrm{p}<0.05$. The a priori power calculation was based on following assumptions: $\mathrm{p}=0.05$ (type 1 error); $\mathrm{P}=0.8$ (type 2 error); coefficient of variation 30\%; smallest significant difference 20\%. This yields at least 36 respondents per group. An additional post-hoc power calculation was done using the actual standard deviations and sampling ratio to determine what level of effect could be found with the 
number of actual respondents. The variability in the judgment of different treatments was analyzed by calculating coefficient of variation (standard deviation divided by the mean). The results are expressed as percentage. The higher the percentage coefficient of variation, the more variability there is between the perceptions of the respondent. Values $<30 \%$ can be considered minor variation.

\section{Ethical considerations}

This survey did not require the approval of the ethical board. All study participants (respondents) were health care professionals and no patients were involved in the study. The patient cases in the questionnaire are hypothetical.

\section{Results}

At the time of the survey, the national Society for the Surgery of the Hand had 95 members. A valid e-mail address was available in the registry for 92 members. A total of 56 surgeons $(61 \%)$ completed the questionnaire. Of the 298 members of the national Society for Hand Therapy, 297 received the questionnaire, and 59 therapists (20\%) completed it. The demographic profile of the participants is summarized in Table 7.

There was a significant difference between the estimate of the benefit of the surgeons judging treatment of patients and the views of the other surgeons judging treatment of themselves concerning carpal tunnel release $(1.8,95 \%$ confidence interval $0.9-2.7 ; 0.2,95 \%$ confidence interval $-0.9-1.5$, respectively) (Figure 4 ). In terms of other procedures, no differences were found. Age and gender had no effect on the responses. Hand surgeons' and hand therapists' perceptions of the benefit of surgery were similar.

Variation of the perceived benefit of operative treatment is summarized in Table 8. The variation ranged from $23 \%$ to $41 \%$ when surgeons were asked to treat patients, 
whereas, when surgeons were asked to treat themselves, the variation was approximately $50 \%$ in carpal tunnel syndrome, dorsal ganglion of the wrist, and boxer's and mallet fractures. Accordingly, there was a trend of more variable opinions, when surgeons were asked to treat themselves.

The functional result after the treatment was regarded as the most important factor determining the treatment of choice in every case (Figure 5). No differences were found between respondent groups.

Using the actual specifications $(\mathrm{p}=0.05, \mathrm{P}=0.8$, sampling ratio $=1.24$, size of the smallest group $=25$, average coefficient of variation $=35 \%$ ) within the responses concerning the benefit of the operative treatment, the sensitivity post-hoc power analysis showed that the minimum detectable effect size for the estimate of the benefit on a 1 -sided level was $26 \%$.

\section{Discussion}

Hand surgeons' perceptions about the benefit of operative treatment for patients was investigated, and it was studied if the perceptions differed if surgeons were considering the same treatment for themselves. Carpal tunnel syndrome was the only hand complaint in which the surgeons' perceptions were inconsistent. Surgeons were less optimistic about the benefit of carpal tunnel release when they considered themselves as patients and there was also more variation in the perceptions. This is interesting because of all surgical procedures of hand, the carpal tunnel release has the highest level of evidence showing that the operation is effective $[1,4]$. Furthermore, the risks related to carpal tunnel release are low [5]. Thus, it would have been plausible that the distribution of the perceived benefit would disperse less (Table 8) - especially as the answers related to flexor tendon repair and thumb replantation cases were very consistent and narrowly distributed, despite lacking evidence about their effectiveness. This suggests 
that customary treatment policies can influence opinions more than scientific evidence.

The agreement between hand surgeons' and hand therapists' views of the benefit of the operative treatment was firm. This contrasts with our hypothesis and previous observations by Alderman et al. [6] who studied the attitudes of hand surgeons and rheumatologists toward surgical operations in patients having rheumatoid arthritis. They found that surgeons were markedly more optimistic about the effectiveness of surgery than rheumatologists. The agreement implies that the clinical perspectives are similar and probably reflect the reality well.

There was no disagreement in the perception of the importance of functional or cosmetic outcomes, cost of the treatment, hospital stay, or length of the sick leave when surgeons were considering treating patients or themselves. Functional result was considered the most important factor, but in fact, little is known about the factors that influence clinical decision-making. Ideally, the physician would inform the patient about the benefits and risks of different treatment modalities and the decision is made together [1]. This includes considering patients' individual values and expectations as well as the current evidence. In practice, many patient- and culture-related characteristics affect clinical decisions (e.g., the tolerance of pain [7] or the disfigurement of the body [8]), while in this study the patients' values or expectations were not included, and the respondents applied their own values and expectations to hypothetical patient cases.

The response rate of hand therapists was low (20\%) when compared to the response rate of surgeons $(61 \%)$. However, the majority of the members of the national Society for Hand Therapy are occupational or physiotherapists who do not work with hand conditions regularly and therefore may have discarded the questionnaire. Thus, it is probable that the 59 therapists who completed the questionnaire are among the most 
competent group of therapist to evaluate the treatment of hand conditions. It must be emphasized that no conclusions regarding the relative benefit of operative treatment between different conditions can be made from the present results, since a case description is a poor proxy for the actual condition. Further, it would be misleading to compare the benefits of treatments of, for example, carpal tunnel syndrome and thumb amputation because the expected benefit is associated more with chosen description of the severity of symptoms or injury. In addition, the effect of expected complication rate and suboptimal recovery was not examined, although this may influence perception of benefit.

In conclusion, we found that surgeons who considered themselves as patients had less confident perception on the benefit of carpal tunnel release compared with surgeons, who considered treating patients. Furthermore, surgeons tended to be more unanimous in their opinions in cases, where there is limited evidence on treatment effect. This suggests that although in theory clinical judgement should be evidence based, personal experience substantially guides the clinical decision making. Hand surgeons and the hand therapists had similar views about the benefits of operative treatment. The most important factor affecting decision-making was the expected functional result.

\section{Acknowledgements}

Mrs. Piia Hautala is thanked for her help in contacting the members of the Finnish Society for Hand Therapy.

\section{Conflict of interest}

The authors declared no potential conflict of interest with respect to the research, authorship, and/or publication of this article. 


\section{References}

1. Schadel-Hopfner M, Windolf J, Antes G, Sauerland S, Diener MK. Evidencebased hand surgery: the role of Cochrane reviews. J Hand Surg Eur Vol. 2008;33:110-7.

2. Ubel PA, Angott AM, Zikmund-Fisher BJ. Physicians recommend different treatments for patients than they would choose for themselves. Arch Intern Med. 2011;171:630-4.

3. Janssen SJ, Teunis T, Guitton TG, Ring D. Do Surgeons Treat Their Patients Like They Would Treat Themselves? Clin Orthop Relat Res. 2015;473:3564-72.

4. Shi Q, MacDermid JC. Is surgical intervention more effective than non-surgical treatment for carpal tunnel syndrome? A systematic review. J Orthop Surg Res. 2011;6:17.

5. Zuo D, Zhou Z, Wang H, Liao Y, Zheng L, Hua Y, Cai Z. Endoscopic versus open carpal tunnel release for idiopathic carpal tunnel syndrome: a metaanalysis of randomized controlled trials. J Orthop Surg Res. 2015;10:12.

6. Alderman AK, Chung KC, Kim HM, Fox DA, Ubel PA. Effectiveness of rheumatoid hand surgery: contrasting perceptions of hand surgeons and rheumatologists. J Hand Surg Am. 2003;28:3-11; discussion 2-3.

7. Rica MA, Norlia A, Rohaizak M, Naqiyah I. Preemptive ropivacaine local anaesthetic infiltration versus postoperative ropivacaine wound infiltration in mastectomy: postoperative pain and drain outputs. Asian J Surg. 2007;30:34-9.

8. Nazerani S, Motamedi MH, Ebadi MR, Nazerani T, Bidarmaghz B. Experience with distal finger replantation: a 20-year retrospective study from a major trauma center. Tech Hand Up Extrem Surg. 2011;15:144-50. 


\section{Legends to figures}

Figure 1. Clinical image provided to the respondents for case 4.

Figure 2. X-ray image provided to the respondents for case 5.

Figure 3. X-ray image provided to the respondents for case 6.

Figure 4. Perception of the benefit of the operative treatment. Minus five stands for "Very Harmful", zero "Neither beneficial nor harmful", and five "Very beneficial". Whiskers indicate $95 \%$ confidence intervals.

Figure 5. Factors affecting decision about the treatment. All respondent groups are pooled. Zero stands for "Not at all important" and four "Very important". Whiskers represent $95 \%$ confidence intervals. 


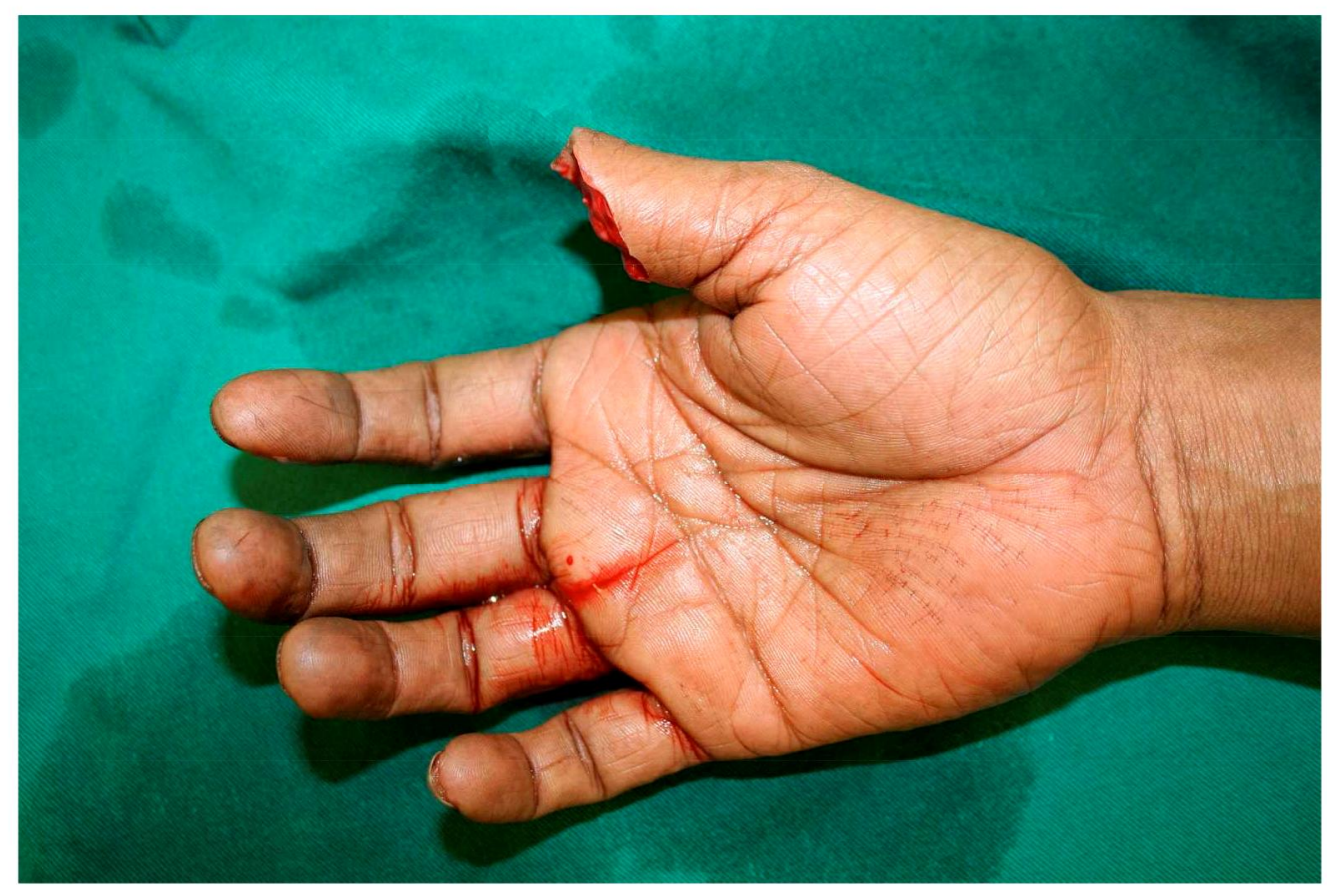

Figure 1. Clinical image provided to the respondents for case 4 .

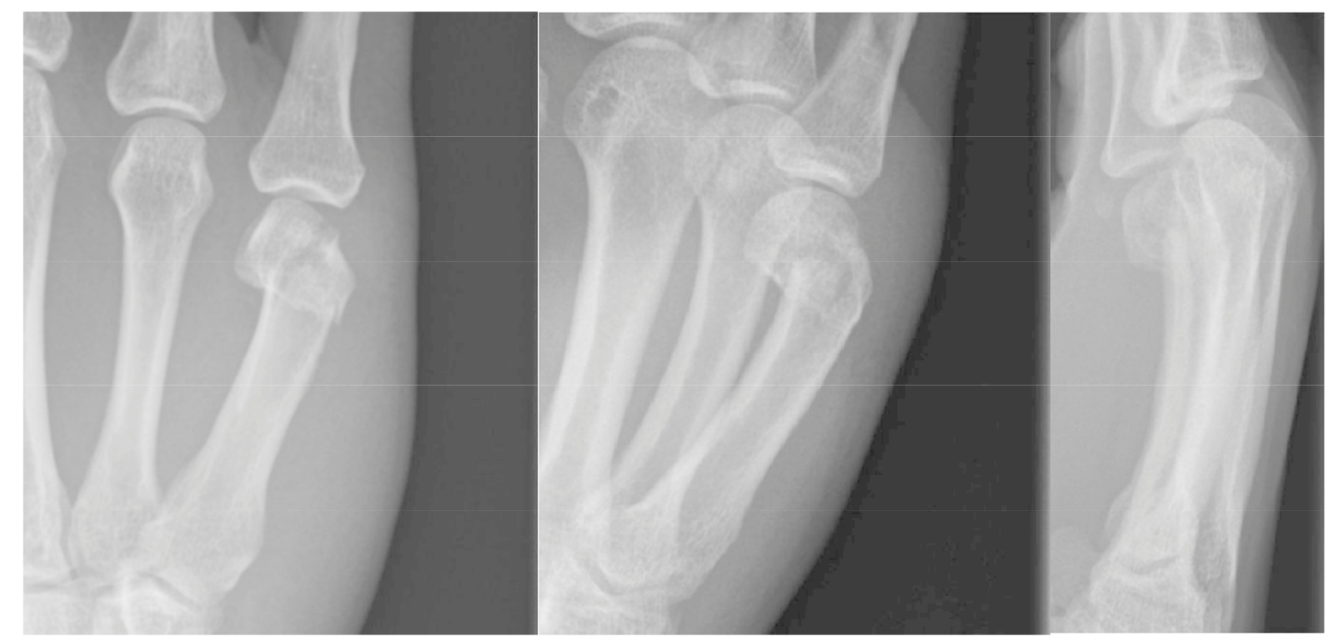

Figure 2. X-ray image provided to the respondents for case 5. 


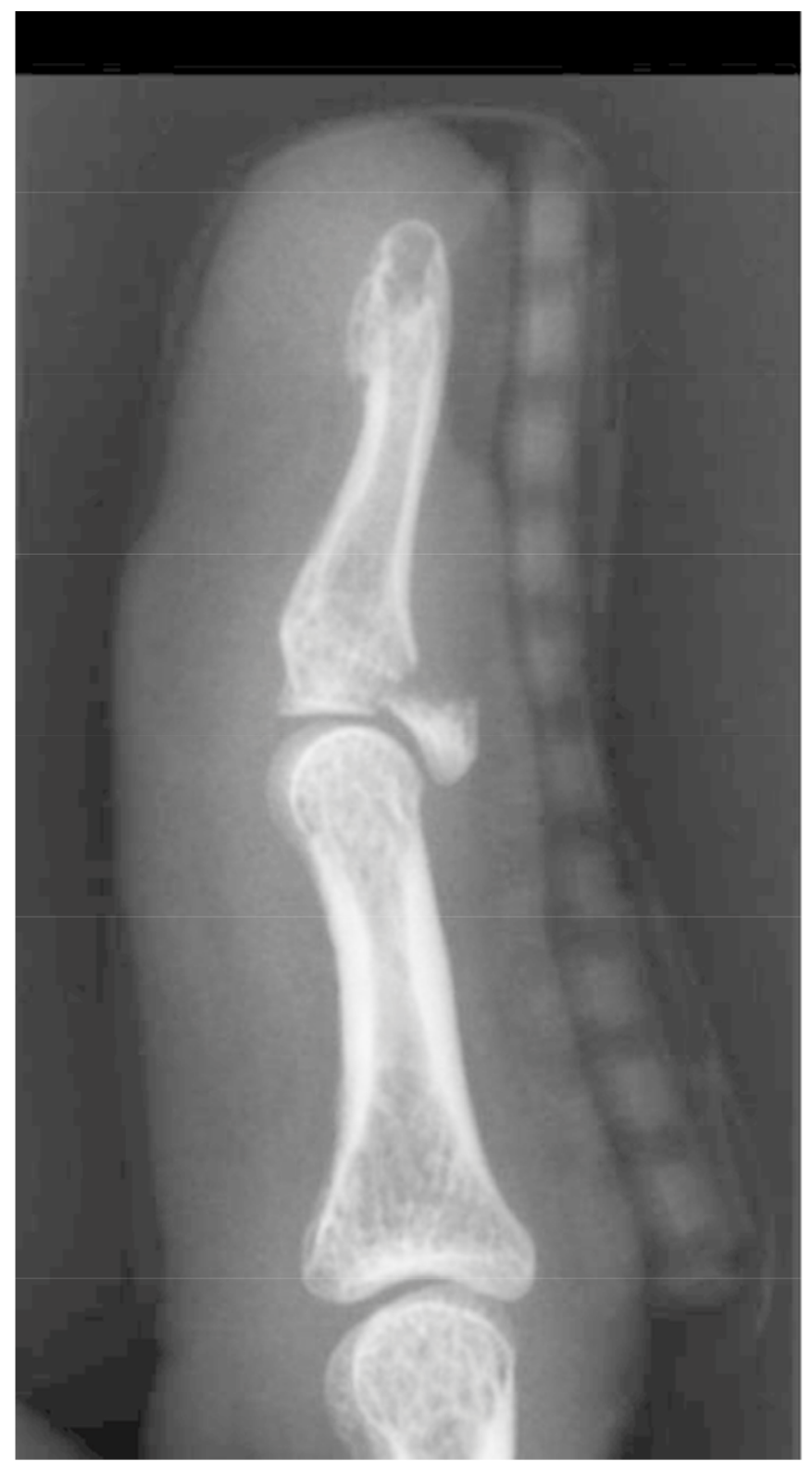

Figure 3. X-ray image provided to the respondents for case 6. 


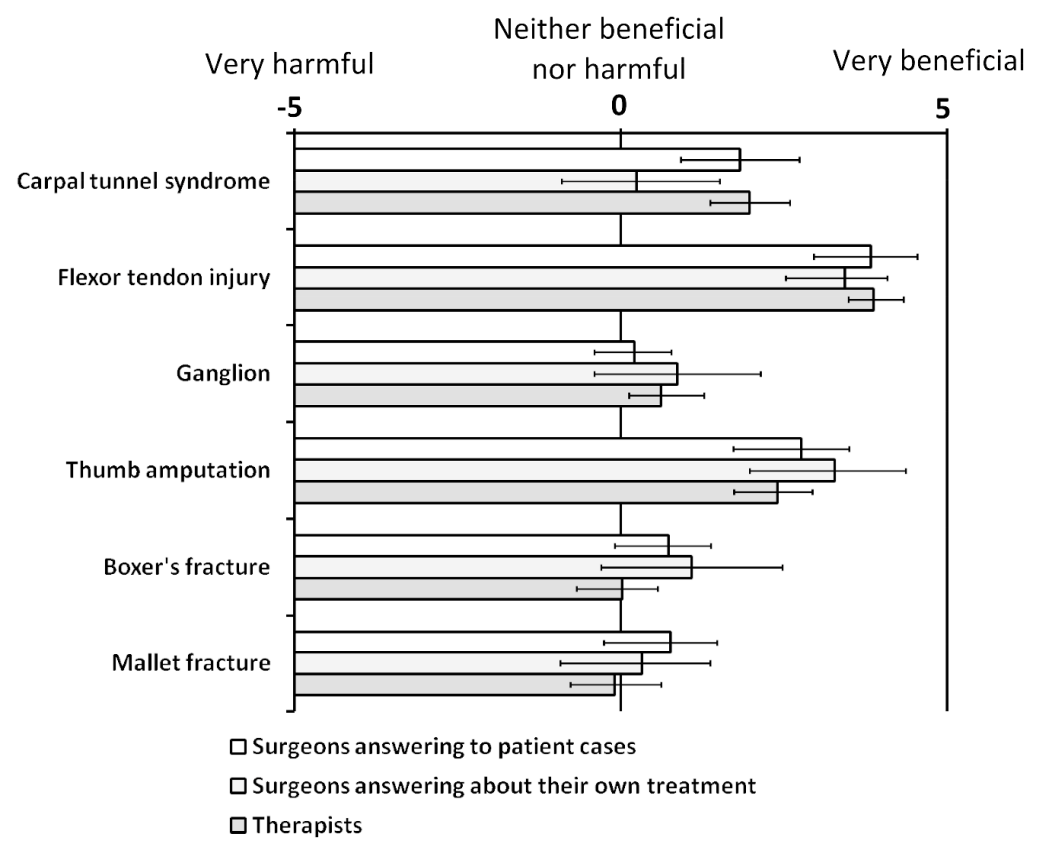

Figure 4. Perception of the benefit of the operative treatment. Minus five stands for "Very Harmful", zero "Neither beneficial nor harmful", and five "Very beneficial". Whiskers indicate $95 \%$ confidence intervals.

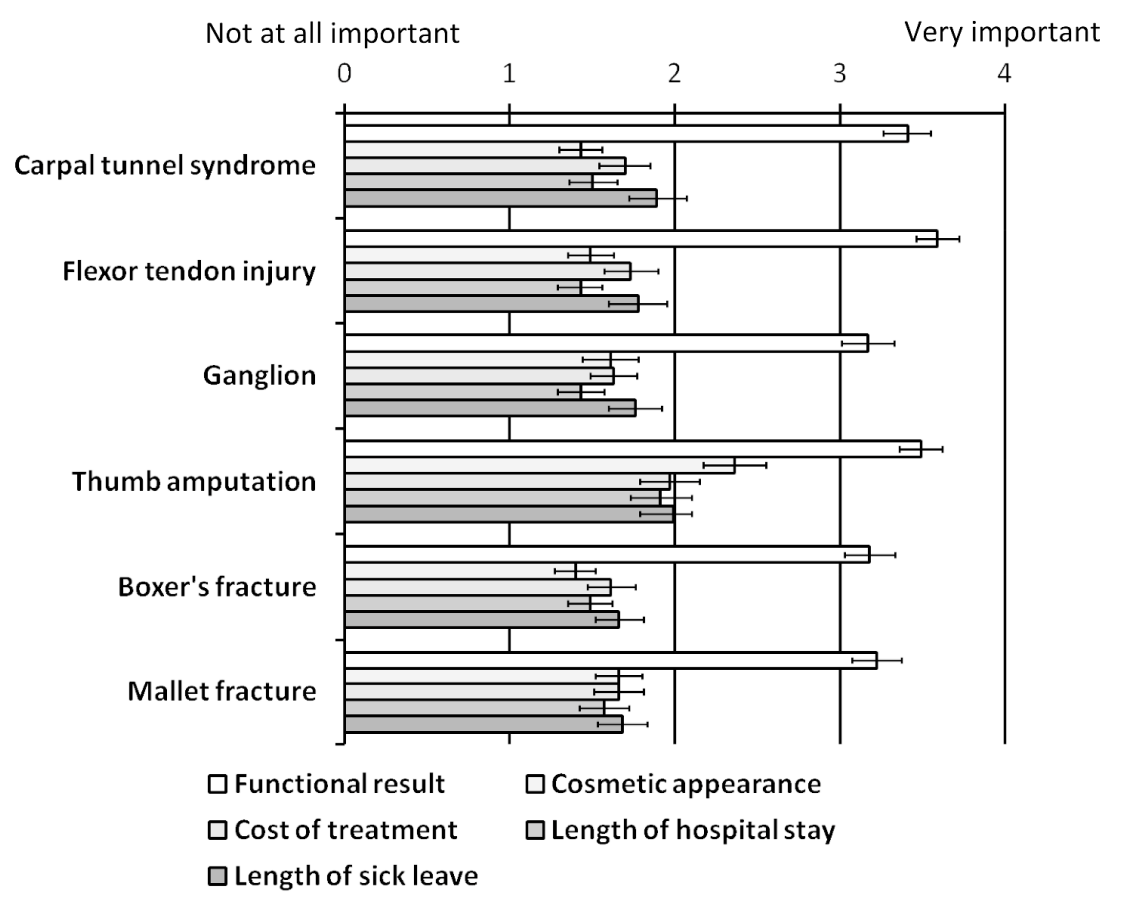

Figure 5. Factors affecting decision about the treatment. All respondent groups are pooled. Zero stands for "Not at all important" and four "Very important". Whiskers represent $95 \%$ confidence intervals. 
Table 1. Patient case with carpal tunnel syndrome (case 1)

\begin{tabular}{ll}
\hline Symptoms: & - Nighttime paresthesias and numbness of the dominant hand. \\
& - Symptoms cause waking up 1-2 times 3-5 nights a week. \\
& - Clumsiness and stiffness of fingers for less than an hour after waking up in \\
& the morning. \\
& - Radiculating pain to forearm may occur occasionally after heavy use. \\
& - No muscle weakness or atrophy. \\
& - Phalen's test positive in 20 seconds. \\
\hline Clinical findings: & - ENG: Median nerve conduction velocity $39 \mathrm{~m} / \mathrm{s}$ over the carpal tunnel \\
& $\begin{array}{l}\text { (laboratory reference value for normal is } 53 \mathrm{~m} / \mathrm{s} \text { ). Motor conduction velocity } \\
\text { and EMG are normal. }\end{array}$ \\
\hline Other findings: & - Open carpal tunnel release. \\
\hline $\begin{array}{l}\text { Proposed surgical } \\
\text { procedure: }\end{array}$ & - Night splinting. \\
\hline Alternative treatment: & - Corticosteroid injection to carpal tunnel. \\
& - Follow up. \\
\hline
\end{tabular}


Table 2. Patient case with flexor tendon injury (case 2).

\begin{tabular}{ll}
\hline History: & - Kitchen knife slipped to the non-dominant index finger causing a volar \\
& wound in proximal interphalangeal joint (PIPJ) level. \\
\hline Symptoms: & - After the accident, the distal interphalangeal joint (DIPJ) of the index \\
& finger has not been actively flexing normally. \\
\hline Clinical findings: & - No active flexor digitorum profundus (FDP) function. \\
& - Flexor digitorum superficialis (FDS) normal function. \\
& - Passive range of motion is normal. \\
& - Sensation is normal. \\
& - Vascular perfusion is normal. \\
\hline Other findings: & - X-ray is normal. \\
\hline Proposed surgical & - Tendon repair and rehabilitation. \\
procedure: & \\
\hline Alternative treatment: & - FDP tenodesis \\
& - DIPJ arthrodesis \\
& - Follow up \\
\hline
\end{tabular}


Table 3. Patient case with dorsal wrist ganglion (case 3).

\begin{tabular}{ll}
\hline Symptoms: & - Pain in the dominant wrist for the past 6 months. \\
& - Pain gets worse when leaning on the hand. \\
& - Sometimes pain in rest, radiculating to the dorsal forearm. \\
\hline Clinical findings: & - 9 mm painful lump palpable in scapholunate-interval in dorsal wrist. \\
& - Active range of motion of the wrist joint is normal. \\
& - Stable SL interval in Watson's test and normal lunotruquetral ballottement \\
& test. \\
& - Full range and pain free motion in the distal radioulnar joint. \\
& - Clinically undisputed ganglion. \\
& - X-ray: normal. \\
\hline Other findings: & - Resection of the ganglion. \\
\hline Proposed surgical & \\
\hline procedure: & - Physiotherapy \\
\hline Alternative treatment: & - Follow up \\
\hline
\end{tabular}


Table 4. Patient case with thumb amputation (case 4).

\begin{tabular}{ll}
\hline History: & - Circular saw injury to the thumb of the dominant hand. \\
& - Amputation across the IP joint in proximal phalanx condylar level. \\
\hline Symptoms: & - Pain. \\
& - Bleeding stopped on the way to the hospital. \\
\hline Clinical findings: & - The amputate is well preserved. \\
& - Irregularity of the wound edges. \\
& - Soft tissue injuries require approximately 4-5 mm shortening of bone. \\
& - Proximal phalanx joint surface is destroyed. \\
& - See Figure 1. \\
\hline Proposed surgical & - Replantation and arthrodesis of the IP-joint. \\
procedure: & \\
\hline Alternative treatment: & - Revision and wound closure. \\
\hline
\end{tabular}


Table 5. Patient case with boxer's fracture (case 5).

\begin{tabular}{ll}
\hline History: & - Fell on outstretched hand in stairs. \\
& - 5th knuckle of the dominant hand hit to stair edge. \\
\hline Symptoms: & - Pain and swelling in the hand. \\
\hline Clinical findings: & - Hematoma and swelling of the 5th metacarpal distal head area. \\
& - No rotatory malalignment. \\
& - Full active range of motion of the IP-joints. \\
\hline Other findings: & - x-ray: 5th metacarpal fracture (Figure 2). \\
\hline Proposed surgical & - Operative reduction and fixation. \\
procedure: & \\
\hline Alternative treatment: & - Splinting or buddy taping. \\
& - Immediate active range-of-motion exercises. \\
& - Follow up. \\
\hline
\end{tabular}


Table 6. Patient case with mallet fracture (case 6).

\begin{tabular}{ll}
\hline History: & - Middle finger of the dominant hand was caught in a closing a door. \\
\hline Symptoms: & - Pain. \\
\hline Clinical findings: & - Dorsal hematoma and swelling in the 3rd DIPJ. \\
& - 20 degrees extension lag in DIPJ. \\
& - PIPJ function normal. \\
\hline Other findings: & - X-ray: Mallet fracture involving 50\% of the joint surface with no apparent \\
& subluxation of the joint (Figure 3). \\
\hline Proposed surgical & - Operative fixation of the fracture. \\
procedure: & \\
\hline Alternative treatment: & - Splinting in extension. \\
& - Follow up. \\
\hline
\end{tabular}


Table 7. Respondent gender, occupation, and age.

\begin{tabular}{|c|c|c|c|}
\hline & $\begin{array}{c}\text { Surgeons } \\
\text { answering to } \\
\text { patient cases, } \\
(\mathrm{n}) \\
\end{array}$ & $\begin{array}{c}\text { Surgeons answering } \\
\text { about their own } \\
\text { treatment, } \\
(\mathrm{n})\end{array}$ & $\begin{array}{l}\text { Therapist, } \\
\text { (n) }\end{array}$ \\
\hline Total & 31 & 25 & 59 \\
\hline Females & 9 & 9 & 57 \\
\hline Males & 22 & 16 & 2 \\
\hline Physiotherapists & - & - & 20 \\
\hline Occupational therapists & - & - & 39 \\
\hline Hand surgeons & 25 & 24 & - \\
\hline Other surgeons & 2 & - & - \\
\hline Hand surgery residents & 4 & 1 & - \\
\hline \multicolumn{4}{|l|}{ Age (years) } \\
\hline$<30$ & 1 & - & 1 \\
\hline $30-39$ & 9 & 7 & 11 \\
\hline $40-49$ & 10 & 8 & 22 \\
\hline $50-59$ & 6 & 6 & 20 \\
\hline $60-69$ & 3 & 2 & 5 \\
\hline$>69$ & 2 & 2 & - \\
\hline
\end{tabular}


Table 8. Coefficient of variation (Standard deviation / Mean, \%) within the judgments about the benefit of operative treatment. Values $<30 \%$ can be considered minor variation.

\begin{tabular}{lccc} 
& $\begin{array}{c}\text { Surgeons } \\
\text { answering } \\
\text { to patient } \\
\text { cases }\end{array}$ & $\begin{array}{c}\text { Surgeons } \\
\text { answering } \\
\text { about their } \\
\text { own } \\
\text { treatment }\end{array}$ & Therapists \\
\hline Carpal tunnel release & 36 & 52 & 33 \\
Flexor tendon repair & 23 & 21 & 18 \\
Ganglion resection & 29 & 50 & 37 \\
Thumb replantation & 30 & 32 & 30 \\
Boxer's fracture fixation & 34 & 53 & 46 \\
Mallet fracture fixation & 41 & 50 & 51 \\
& & & \\
\hline
\end{tabular}

